IPPP $/ 10 / 27, \mathrm{DCPT} / 10 / 54$

\title{
Regular Wilson loops and MHV amplitudes at weak and strong coupling
}

\author{
Paul Heslop ${ }^{a}$ and Valentin V. Khoze ${ }^{b}$ \\ a Institute for Particle Physics Phenomenology, \\ Department of Mathematical Sciences and Department of Physics \\ Durham University, Durham, DH1 3LE, United Kingdom \\ ${ }^{b}$ Institute for Particle Physics Phenomenology, \\ Department of Physics, Durham University, \\ Durham, DH1 3LE, United Kingdom
}

\begin{abstract}
Traditionally, the duality between Wilson loops and amplitudes beyond one loop in $\mathcal{N}=4 \mathrm{SYM}$ is characterised by the remainder function. Because of the perturbative origins of the BDS expression, the remainder function is more natural at weak than at strong coupling. We advocate instead a more direct approach, based on considering ratios of Wilson loops. This allows us to define a manifestly finite, regularisation independent, conformally invariant quantity. It does not make a direct reference to the BDS expression and the definition is regularisation independent. It is a natural object at weak and at strong coupling, and in the latter case is directly related to the free energy of an auxiliary integrable system. We then compute these ratios for continuous families of regular polygons for 6,8 and 10 points at one and two-loops. These results are compared to expressions derived recently at strong coupling.
\end{abstract}

\{paul.heslop, valya.khoze\}@durham.ac.uk 


\section{Introduction}

It has been conjectured [1-3] that in planar $\mathcal{N}=4$ super Yang-Mills (SYM) there is a non-trivial relation between scattering amplitudes and Wilson loops,

$$
W_{n}:=W\left[\mathcal{C}_{n}\right]=\operatorname{Tr} \mathcal{P} \exp \left[i g \oint_{\mathcal{C}_{n}} d \tau \dot{x}^{\mu}(\tau) A_{\mu}(x(\tau))\right],
$$

with a lightlike $n$-edged polygonal contour $\mathcal{C}_{n}$ obtained by attaching the momenta of the scattered particles $p_{1}, \ldots, p_{n}$ one after the other, following the order of the colour generators in the colour-ordered scattering amplitude. The vertices, $x_{i}$, of the polygon are related to the external momenta via $p_{i}=x_{i}-x_{i+1}$, where $x_{n+1}=x_{1}$. There has been an increasing amount of evidence in support of this amplitude/Wilson loop duality relation $[1-8]$.

For MHV amplitudes, $\mathcal{A}_{n}^{\mathrm{MHV}}=\mathcal{A}_{n}^{\mathrm{MHV} \text { tree }} \times \mathcal{M}_{n}$, the MHV amplitude/Wilson loop duality [1 3] at one loop states simply that $\mathcal{M}_{n}^{(1)}=W_{n}^{(1)}+$ const [3], whereas beyond one loop it is normally understood in terms of the remainder function. The remainder function of the amplitude $\mathcal{R}_{n}$ (or of the Wilson loop $\mathcal{R}_{n}^{W L}$ ) is defined as the difference between the logarithm of the entire amplitude $\mathcal{M}_{n}$ (Wilson loop $W_{n}$ ) and the known BDS expression obtained in [9, 10], so that

$$
\begin{aligned}
\mathcal{R}_{n} & =\log \left(\mathcal{M}_{n}\right)-(B D S)_{n} \\
\mathcal{R}_{n}^{W L} & =\log \left(W_{n}\right)-(B D S)_{n}^{W L} .
\end{aligned}
$$

The BDS expressions for both the amplitude and the Wilson loop can be found in the Appendix where we also outline the difference between the amplitude and Wilson loop expressions. The duality then states that the two remainder functions are identical [6] 8] (and in particular no constant shifts are allowed)

$$
\mathcal{R}_{n}=\mathcal{R}_{n}^{W L}
$$

The amplitudes and Wilson loops are themselves divergent quantities. The amplitudes contain infrared and the Wilson loops ultraviolet divergences. These divergences break the (dual) conformal symmetry of the theory. The remainder function however is a quantity which is constructed to be manifestly finite since the divergences are cancelled by the BDS contributions. Furthermore, the remainder functions are known to be conformally invariant and as such they depend on the kinematics only through the conformal cross-ratios $u_{i j}$. 1

\footnotetext{
${ }^{1}$ More precisely the Wilson loop remainder is invariant under conformal transformations in $\mathcal{N}=4$ super Yang-Mills [2]. Assuming the Wilson loop/amplitude duality (1.3), the amplitude remainder function must then also only depend on conformal cross-ratios. This has become known as the dual conformal invariance of the amplitude.
} 
The BDS expressions are essentially determined by the one loop amplitudes and as such the first non-vanishing contribution to the remainder function appears at two loops. Even though BDS expressions have their origin at one loop in perturbation theory, they do depend on the coupling a through cusp anomalous dimensions (as recalled in the Appendix) and are straightforwardly extended to all values of the coupling. Thus the remainder functions in (1.3) are defined for all values of the coupling, and in particular can be constructed at strong or at weak coupling. Traditionally computations for Wilson loops or amplitudes beyond one loop have always been interpreted in terms of the remainder function at weak and at strong coupling.

However, the one-loop perturbative origin of the BDS expression means that the remainder function is not the most natural quantity appearing at strong coupling. We would like to formulate an approach which does not involve $\mathcal{R}_{n}$ and that allows for a more direct comparison of weak and strong coupling results. In the following section we will define such a quantity in terms of a ratio of a Wilson loop with an appropriately defined reference Wilson loop. We will further argue that this object is conformally invariant and provides a natural formulation of the Wilson loop/amplitude duality.

We will then compute this finite conformal ratio for hexagons, octagons and decagons. For concreteness and to keep the kinematics manageable we will concentrate on families of regular polygons. Strong coupling computations for these regular polygons were computed very recently in [11. We will compare and supplement these strong coupling answers with one-loop and two-loop results.

In section 3 we define the regular kinematics, in section 4 we present the results for the hexagons, sections 5 and 6 contain our analysis of regular octagons and decagons.

\section{The finite conformally invariant ratio}

At strong coupling, $a \rightarrow \infty$, the quantity of interest is $\sqrt{2 a} A$ where $A$ is the area of a world sheet ending on the polygonal Wilson loop [1]. The area is infinite (which is a reflection of the divergences of the amplitude/Wilson loop) and needs to be regularised. However we do not wish to rely on any specific scheme (for example dimensional regularisation which is the standard choice made in weak coupling computations is not natural at strong coupling and not what is used in practice there [11-14]). Thus we will construct a manifestly finite quantity, which should be independent of the regularisation used.

In general the area is a Lorentz invariant quantity which depends on two-particle invariants, $s_{i}=\left(p_{i}+p_{i+1}\right)^{2}$, and multi-particle invariants, $t_{i}^{[r>2]}=\left(p_{i}+p_{i+1}+\cdots+\right.$ 
$\left.p_{i+r-1}\right)^{2}$. It can be represented as 12

$$
A(s, t)=A_{\text {cutoff }}(s)+A_{\text {finite }}(s, t), \quad A_{\text {cutoff }}(s)=4 \int_{\Sigma_{0}, z_{\mathrm{AdS}}>\epsilon_{c}} d^{2} w .
$$

Here $\Sigma_{0}$ is an appropriate simplified surface in $A d S_{5}$ and $\epsilon_{c}$ is the cutoff in the radial direction. The important point for us is that $A_{\text {cutoff }}$ depends on the kinematics only through the two-particle invariants $s_{i}$. In particular [11-13],

$$
A_{\text {cutoff }}=\frac{1}{8} \sum_{i=1}^{n}\left(\log \epsilon_{c}^{2} s_{i}\right)^{2}-\frac{1}{8} \sum_{i=1}^{n}\left(\left(\log s_{i}\right)^{2}+\sum_{k=0}^{(n-2) / 4}(-1)^{k+1} \log s_{i} \log s_{i+1+2 k}\right)
$$

for $n$ even, and a very similar formula holds for odd values of $n$,

$$
A_{\text {cutoff }}=\frac{1}{8} \sum_{i=1}^{n}\left(\log \epsilon_{c}^{2} s_{i}\right)^{2}-\frac{1}{4} \sum_{i=1}^{n}\left(\left(\log s_{i}\right)^{2}+\sum_{k=0}^{2 K}(-1)^{k+1} \log s_{i} \log s_{i+1+2 k}\right)
$$

where $n=4 K+1$ or $n=4 K+3$.

We can thus consider the difference between the area $A(s, t)$ and a reference area $A(s, \tilde{t})$ which has the same values of two-particle invariants, whilst the multi-particle invariants $\tilde{t}$ are fixed. The divergent contributions, $A_{\text {cutoff }}$, cancel and the difference between the two areas is manifestly finite.

This cancellation of divergences in the difference between the area- and the reference area-like quantities is a general feature which holds at strong and at weak coupling to all orders in perturbation theory, both for the scattering amplitudes and the Wilson loops. In the context of Wilson loops, the difference of areas is simply the logarithm of the ratio between the two Wilson loops,

$$
\log \left(\frac{W_{n}}{W_{n}^{\mathrm{ref}}}\right):=w_{n}(s, t)-w_{n}^{\mathrm{ref}}(s, \tilde{t})=\text { finite } .
$$

This is a finite quantity at weak coupling as well, since all the divergences of each $w_{n}$, in dimensional regularisation $D=4-2 \epsilon$, are of the form (see Appendix)

$$
-\frac{1}{2 \epsilon^{2}} \sum_{L=1}^{\infty} a^{L} \frac{f^{(L)}(\epsilon)}{L^{2}} \sum_{i=1}^{n}\left(\frac{-s_{i}}{\mu^{2}}\right)^{-L \epsilon}
$$

ie they depend only on two-particle invariants and thus cancel between the two terms on the right hand side of (2.4). Exactly the same argument holds in the amplitudes case for $\log \left(\mathcal{M}_{n} / \mathcal{M}_{n}^{\text {ref }}\right)$. Indeed in any regularisation the divergences will depend only on two-particle invariants 2 , and hence this argument is independent of any specific regularisation scheme.

\footnotetext{
${ }^{2}$ All infrared divergences of colour-ordered scattering amplitudes at large $N$ arise from the $\mathrm{Su}$ dakov form-factors associated to each consecutive pair of external legs.
} 
To proceed, we note that the number of independent multi-particle invariants $t_{i}^{[r]}$ is $n(n-5) / 2$, precisely the same as the number of independent cross-ratios $u_{i j}$ defined as

$$
u_{i j}=\frac{x_{i j+1}^{2} x_{i+1 j}^{2}}{x_{i j}^{2} x_{i+1 j+1}^{2}},
$$

where $x_{i}$ are the vertices of the polygonal contour of the Wilson loop. This matching holds when we do not impose the Gramm determinant constraints, ie the external momenta are not restricted to four dimensions [8] 3. Furthermore, as long as the number of external particles (or for the Wilson loop the number of edges) $n$ is not divisible by four we can trade all multi-particle invariants for the cross-ratios $\{s, t\} \rightarrow$ $\{s, u\}$. Thus the area can be recast as a function of $s_{i}$ and $u_{i j}$.

In the special case when $n=4 K$, some of the cross-ratios depend only on twoparticle invariants and a complete separation between the $s$-variables and $u$-variables is not possible. This case will be treated separately in section 5 where we will show that special kinematical subsets for $n=4 K$ can still be treated in essentially the same way. Until then we will concentrate on cases with $n$ not divisible by four and adopt the $\{s, u\}$ basis.

The next point to note is that in the $\{s, u\}$ basis the second term in the first equation in (2.1) does not depend on the two-particle invariants, $A_{\text {finite }}=A_{\text {finite }}(u)$. This is a consequence of the fact [12] that the second term on the right hand side of (2.2) (or (2.3) for odd values of $n$ ) is a solution of the anomalous Ward identities for broken conformal invariance [2]. Since the entire area (or more precisely the finite area which excludes the explicit cut-off $\epsilon_{c}$-dependence) must also satisfy the same conformal anomaly equation, any finite correction to the second term in (2.2) must be a solution to the corresponding homogeneous equation. $A_{\text {finite }}$ is precisely the finite part of the area missed by the cut-off contribution, it must be a solution to the homogeneous equation, and as such is a function solely of the cross-ratios $u_{i j}$. In Refs. [11-13] $A_{\text {finite }}(u)$ was itself represented as the sum of a few terms, $A_{\text {finite }}=$ $A_{\text {free }}+A_{\text {periods }}+A_{\text {extra }}$ each of which was derived from independent reasoning and with particular attention payed to the free energy contribution determined through the Thermodynamic Bethe Ansatz (TBA) equations. At present it is not known if and how the TBA structure can arise in the opposite weak coupling limit, thus in this paper we shall not attempt to break $A_{\text {finite }}$ into separate master functions at

\footnotetext{
${ }^{3}$ Conformal invariance of the remainder function indeed did not require imposing the Gramm determinant constraint $[\underline{6}$.

${ }^{4}$ In this sense this contribution is the strong-coupling analogue of the BDS expression which is also a particular solution of the anomaly equations; it was termed 'BDS-like' in [12. For $n=4,5$ the BDS and the BDS-like expressions are identical, but for general $n \geq 6$ they are different, in particular BDS depends on multi-particle invariants while BDS-like does not. For $n \neq 4 K$ BDSlike is the unique solution of this anomaly equation which depends on the kinematics only through two-particle invariants.
} 
weak coupling and instead concentrate on the $u$-dependence of the entire area. It is worthwhile pointing out however, that for the $Z_{n}$-symmetric Wilson loops (which are the main computational application in this paper), the strong coupling limit of $\log \left(W_{n} / W_{n}^{\text {ref }}\right)$ is equivalent to the free-energy contribution, $A_{\text {free }}$. The remaining terms, $A_{\text {periods }}$ and $A_{\text {extra }}$, will not contribute to this ratio in the $Z_{n}$-symmetric case. In other words, the quantity $\log \left(W_{n} / W_{n}^{\text {ref }}\right)$ will allow one to zoom in on the analogue of the the free-energy contribution at weak coupling.

It follows that the difference between the area and the reference area,

$$
A(s, u)-A(s, \tilde{u})=A_{\text {finite }}(u)-A_{\text {finite }}(\tilde{u}) \equiv f(u, \tilde{u})
$$

is a finite and conformally-invariant function in the sense that it depends on the kinematics only through the conformal cross-ratios $u_{i j}$ as well as the reference ratios $\tilde{u}_{i j}$ which we view as fixed. Manifest finiteness of the area difference also implies that it can be computed in any regularisation scheme.

Note that exactly the same reasoning applies, in general, not only to the strongcoupling regime, but also at weak coupling to all orders in perturbation theory and both for scattering amplitudes and Wilson loops. For Wilson loops, as we already discussed, the relevant quantity is the logarithm of the ratio between the two Wilson loops,

$$
\log \left(\frac{W_{n}}{W_{n}^{\mathrm{ref}}}\right):=w_{n}(s, u)-w_{n}^{\operatorname{ref}}(s, \tilde{u})=\operatorname{finite}(u, \tilde{u}) .
$$

Once again the right-hand side is a function of conformal cross-ratios only. This is ensured by the fact that $w_{n}(s, u)$ satisfies anomalous conformal Ward identities a particular solution of which is (2.2), (2.3) which crucially depends only on two-particle invariants. Thus the difference of two w's with the same two-particle invariants satisfies non-anomalous conformal Ward identities and as such is a function of crossratios only.

The Wilson loop/MHV amplitude duality then takes a remarkably simple form in terms of the ratio. It is the statement that

$$
\log \left(\frac{W_{n}}{W_{n}^{\text {ref }}}\right)(u, \tilde{u})=\log \left(\frac{\mathcal{M}_{n}}{\mathcal{M}_{n}^{\text {ref }}}\right)(u, \tilde{u}),
$$

or even more simply

$$
\frac{W_{n}}{W_{n}^{\text {ref }}}=\frac{\mathcal{M}_{n}}{\mathcal{M}_{n}^{\text {ref }}}
$$

where we treat the reference variables $\tilde{u}$ as fixed. This duality correspondence is in terms of manifestly finite, conformally-invariant quantities. It is defined in a 
regularisation-independent manner $5^{5}$ ) and is expected to hold at all values of the coupling constant including, of course, one loop where it is a non-trivial statement (unlike the formulation in terms of $\mathcal{R}$ ).

We would like to add a word of caution in respect to what is meant by the conformal invariance of (2.8)-(2.10). The right hand side of equation (2.8) is conformally invariant after all the dependence on the two-particle invariants is cancelled between $W_{n}$ and $W_{n}^{\text {ref }}$. However, the actual ratio was constructed in a given frame where we chose the $s$-variables in $W_{n}^{\text {ref }}(s, \tilde{u})$ to be the same as in the original Wilson loop $W_{n}(s, u)$. If one performed a conformal transformation on the individual Wilson loops before computing their ratio, the $s$-variables would no longer match.

Another interesting observation is that we were free to remove the log in (2.9) without any loss of information because the ratio is finite. This should be compared with the amplitude/Wilson loop itself and its log (keeping, in both cases, only terms up to $\left.O\left(\epsilon^{0}\right)\right)$ which contain different pieces of information. The reason for this is that $1 / \epsilon^{2}$ contributions in the singular part of the exponent can hit $O(\epsilon)$ pieces resulting in $1 / \epsilon$ contributions. These can not be seen from the log of the amplitude itself. For example the five-point two loop amplitude contains parity odd pieces at $O(1 / \epsilon)$ whereas in the $\log$ of the amplitude they cancel and appear only at $O(\epsilon)[16]^{6}$. The ratio of the amplitudes is however free from such effects. Interestingly, it was pointed out in [15] that amplitudes in the Coulomb branch regularisation are also free from such effects. Our ratio is of course defined independently of the regularisation in the first place.

For generic non-MHV amplitudes in $\mathcal{N}=4$ SYM the quantity

$$
\frac{\mathcal{A}_{n}^{\mathrm{NMHV}}(s, t, h)}{\mathcal{A}_{n}^{\mathrm{ref}}(s, \tilde{t}, \tilde{h})}
$$

is a finite quantity 7 . Here $h, \tilde{h}$ denote helicities and particle types. Indeed, it has a nice interpretation as the ratio of hard amplitudes. Infrared factorisation arguments [18 21, dictate that any amplitude is a product of soft, jet and hard amplitudes, where the hard amplitude is an infrared safe quantity. In planar perturbation theory (where we work) the jet times soft amplitude becomes simply a product of (square roots of) Sudakov form-factors and as such they depend only on two-particle invariants. What survives in the ratio is the ratio of hard amplitudes.

\footnotetext{
${ }^{5}$ For example one can compute the ratio $\mathcal{M}_{n} / \mathcal{M}_{n}^{\text {ref }}$ on the Coulomb phase of $\mathcal{N}=4 \mathrm{SYM}$ where infrared divergences are regulated by the masses as explained in [15].

${ }^{6}$ Subsequently this has also been seen in the high energy regime in [17].

${ }^{7}$ Indeed this quantity should be infrared finite in any large $N$ gauge theory.
} 
If we factor out the corresponding tree level amplitude, then the object

$$
\frac{\left(\mathcal{A}_{n}^{\text {NonMHV }} / \mathcal{A}_{n}^{\text {NonMHV tree }}\right)(s, u, h)}{\left(\mathcal{A}_{n}^{\text {ref }} / \mathcal{A}_{n}^{\text {ref tree }}\right)(s, \tilde{u}, \tilde{h})} .
$$

is not only finite but we expect it to be (dual) conformally invariant in view of the expected dual conformal properties of non-MHV amplitudes [22 24]. Normalisation of all amplitudes by the tree-level factor also naturally fits with the proposal [25] that in the strong coupling regime any generic (MHV or NonMHV) amplitude is the product of the corresponding tree-level amplitude and the helicity-independent exponential factor involving the same semiclassical action (area) as the one found for MHV amplitudes in [1]. Of course the Wilson loop dual for non-MHV amplitudes is not known at present.

In the rest of the paper we will concentrate on the ratio of Wilson loops (2.8) in $\mathcal{N}=4$ SYM at large $N$. In the next section we will define the kinematics for the continuous families of regular polygons which will be needed for our applications.

\section{A Note on multi-collinear limits}

One nice property of the remainder function is its very simple transformation properties under collinear limits [8]

$$
\mathcal{R}_{n} \rightarrow \mathcal{R}_{n-k}+\mathcal{R}_{k+4}
$$

for a $(k+1)$-collinear limit, ie where $(k+1)$ momenta become collinear. This equation is plotted in figure 1. The first term on the right-hand side is the reduced $n-k$ polygon emerging in this multi-collinear limit. Meanwhile the second term arises from the $(k+1)$-collinear splitting function 8 . Note that the latter is present because the collinear limit is taken after expanding the full Wilson loop in $\epsilon$ (that is the distance between the $k$ vertices and the dotted line is limited by the UV cutoff.)

It is a nice feature that both terms on the right-hand side are themselves remainder functions of different ranks. In this multi-collinear limit, the entire set of cross-ratios of $\mathcal{R}_{n}$ decomposes into the set of cross-ratios for $\mathcal{R}_{n-k}$ and the set of cross-ratios for $\mathcal{R}_{k+4}$. These two sets can be most easily determined from figure 1, In particular one draws all independent $u$-cross-ratios correspnding to the first polygon on the righthand side for the first set, and all independent cross-ratios within the second polygon for the second set. The first set is independent of the muti-collinear momenta whereas the second set depends only on the multi-collinear variables $\left(z_{1}, \ldots z_{k+1}\right.$ and the ratios of vanishing kinematic invariants.)

\footnotetext{
${ }^{8}$ More precisely the part of the splitting function not already contained in the BDS expression.
} 
For example when $k=2$ we have a triple collinear limit. We choose $p_{4}, p_{5}$ and $p_{6}$ to be the collinear momenta, so that

$p_{4}:=x_{4}-x_{5}=z_{1} P, \quad p_{5}=x_{5}-x_{6}=z_{2} P, \quad p_{6}=x_{6}-x_{1}=z_{3} P, \quad z_{1}+z_{2}+z_{3}=1$, and $\mathcal{R}_{k+4}=\mathcal{R}_{6}\left(\bar{u}_{1}, \bar{u}_{2}, \bar{u}_{3}\right)$ where [6, 8 ]

$$
\bar{u}_{1}=\frac{1}{1-z_{3}} \frac{s_{45}}{s_{456}}, \quad \bar{u}_{2}=\frac{1}{1-z_{1}} \frac{s_{56}}{s_{456}}, \quad \bar{u}_{3}=\frac{z_{1} z_{3}}{\left(1-z_{1}\right)\left(1-z_{3}\right)} .
$$
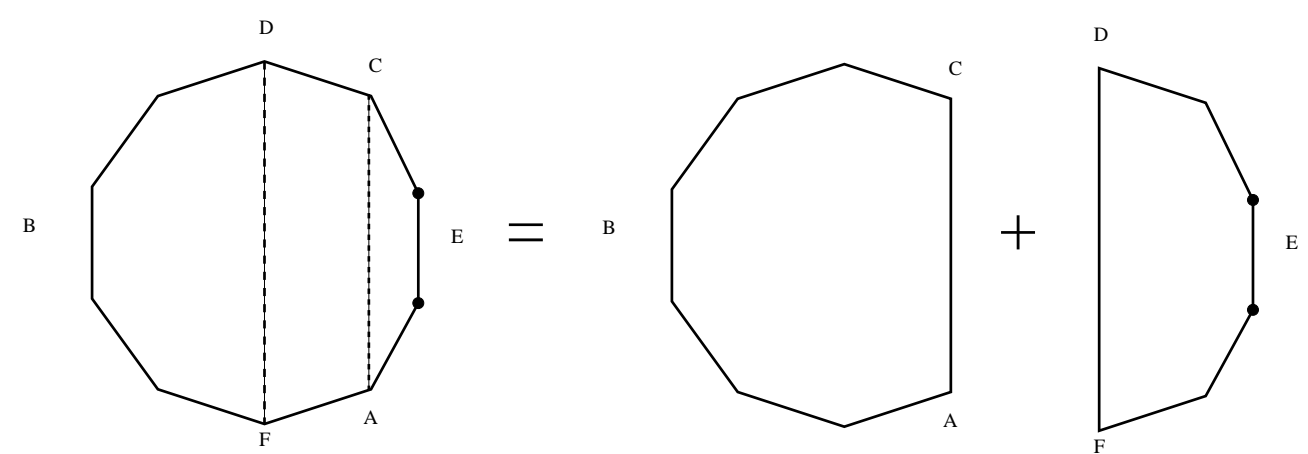

Figure 1: Multi-collinear limit. Here we represent the decomposition in (2.13). The thick dots represent the $k$ vertices which approach the dashed line AC in the $(k+1)$ collinear limit.

A very similar property holds for the ratio of Wilson loops (Amplitudes) defined here too, with the caveat that one needs to keep both variables, $u$ and $\tilde{u}$ active as the reference cross-ratios $\tilde{u}$ do change under collinear limits. Thus we have $w_{n}(s, u)-$ $w_{n}^{\mathrm{ref}}(s, \tilde{u}) \rightarrow\left(w_{n-k}(s, u)-w_{n-k}^{\mathrm{ref}}(s, \tilde{u})\right)+\left(w_{k+4}(s, \bar{u})-w_{k+4}^{\mathrm{ref}}(s, \overline{\tilde{u}})\right)$. For the original and the reference Wilson loop to satisfy the same collinear limits, the cross-ratios in the splitting functions $\bar{u}$ and $\overline{\tilde{u}}$ must of course be the same functions of the multi-collinear variables, $z_{1}, \ldots z_{k}$ etc. Thus $\bar{u}=\overline{\tilde{u}}$ and

$$
w_{n}(s, u)-w_{n}^{\mathrm{ref}}(s, \tilde{u}) \rightarrow w_{n-k}(s, u)-w_{n-k}^{\mathrm{ref}}(s, \tilde{u}) .
$$

Thus for the ratio, the collinear limit is purely geometrical with no contributions from the splitting function as you might expect for a physical and finite quantity 9 .

\section{A continuous family of regular polygons}

In general at $n$-points the number of independent cross-ratios, $n(n-5) / 2$, grows quadratically with $n$. It is therefore useful to identify smaller subsets on this con-

\footnotetext{
${ }^{9}$ In distinction with the standard definition of the remainder function which included subtraction of the BDS term expanded in $\epsilon$, here the collinear limit can be taken with or without expanding in any UV regulator.
} 
figurations space, guided by symmetry. One example of such a symmetry is cyclic symmetry, $Z_{n}$, which singles out regular polygons.

The authors of [11] argued that for polygons in four dimensions, there is a one parameter family 10 of regular polygons for any even $n$. The family depends continuously on the parameter $\phi$ and varying $\phi$ one covers a particular slice of the $u_{i j}$ space. In particular $\phi=0$ and $\phi=(n-4) \pi / 2$ correspond to special regular polygons, which lie entirely in 3 and 2 dimensional subspaces respectively.

We will now briefly discuss how one can introduce general $Z_{n}$ symmetric kinematics. To this end we first need to identify a conformal transformation $T$ such that $T^{n}=1$. The vertices of the $Z_{n}$ symmetric polygon $X^{(p)}$ are obtained by applying $T$ consecutively $p$ times to an arbitrary point $X^{(0)}$ so that $X^{(p)}=T^{p} X^{(0)}$. A natural way to discuss the action of the conformal group $O(2,4)$ in Minkowski space, is to describe Minkowski space in terms of six projective co-ordinates $X_{I}$ living in $2+4$ dimensions satisfying

$$
X_{-1}^{2}+X_{0}^{2}-X_{1}^{2}-X_{2}^{2}-X_{3}^{2}-X_{4}^{2}=0 .
$$

The conformal group $O(2,4)$ then acts linearly on these coordinates and four-dimensional Minkowski space coordinates can be obtained straightforwardly from these via (3.8).

By choosing a suitable basis, a general element of $O(2,4)$ can be represented in terms of three $2 \times 2$ blocks $M_{1}, M_{2}, M_{3}$ which are either rotations or reflections acting on two coordinates each. For this to satisfy $T^{n}=1$ each of the $O(2)$ rotations must be characterised by an angle $2 \pi r / n$ with $r$ an integer and reflections can only be introduced for $n$ even. For example a $Z_{n}$ element composed of two rotations and one reflection acting on $X$-space is given by

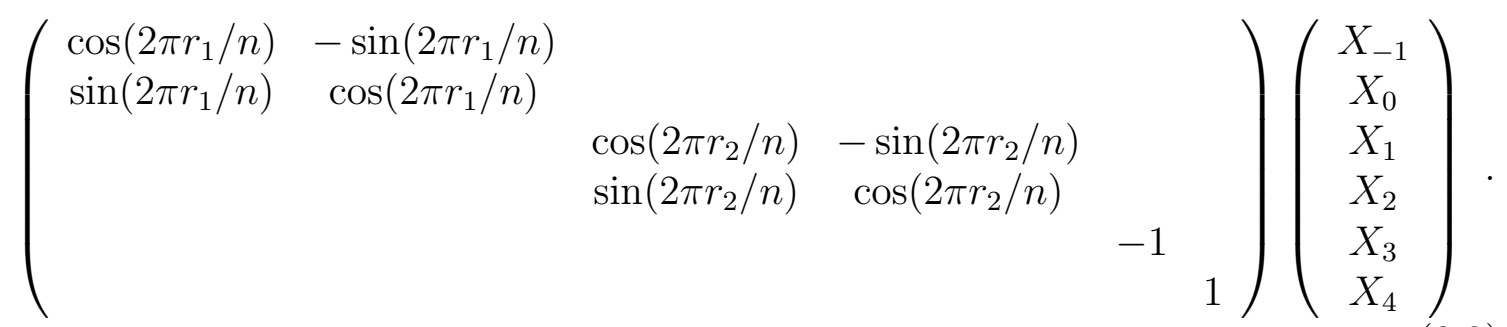

The particular configuration of [11] corresponds to the equation above with $r_{1}=1$ and $r_{2}=2$. In this case with two rotations and one reflection, without loss of generality we can choose our initial vertex $X^{(0)}$ as

$$
\left(X^{(0)}\right)^{T}=\left(l_{3}, 0, l_{2}, 0, l_{1}, 1\right) .
$$

\footnotetext{
${ }^{10}$ Allowing external particles to lie in an arbitrary number of dimensions gives higher dimensional families of regular polygons, for example at 8 points we would have a two parameter family and at 10 points a three parameter family.
} 
From now on we will concentrate on this specific $Z_{n}$-symmetric family with $r_{1}=1$ and $r_{2}=2$ and postpone the Wilson loop computations of other regular configurations arising from this setup to future work.

Applying the transformation (3.2) consecutively on (3.3) we obtain the polygon vertices

$$
\begin{array}{ll}
X_{-1}^{(p)}=l_{3} \cos \left(\frac{2 \pi p}{n}\right) & X_{0}^{(p)}=l_{3} \sin \left(\frac{2 \pi p}{n}\right) \\
X_{1}^{(p)}=l_{2} \cos \left(\frac{4 \pi p}{n}\right) & X_{2}^{(p)}=l_{2} \sin \left(\frac{4 \pi p}{n}\right) \\
X_{3}^{(p)}=(-1)^{p} l_{1} & X_{4}^{(p)}=1 .
\end{array}
$$

where $p=1 \ldots n$ labels the $n$ vertices. Eq (3.1) implies that $l_{1}, l_{2}$ and $l_{3}$ satisfy

$$
1+l_{1}^{2}+l_{2}^{2}-l_{3}^{2}=0 .
$$

So far the discussion applies to arbitrary regular polygons. We are specifically interested in null polygons for which $X^{(p)} \cdot X^{(p+1)}=0$ giving the additional constraint

$$
l_{1}^{2}+\sin \left(\frac{2 \pi}{n}\right)^{2} l_{2}^{2}-\sin \left(\frac{\pi}{n}\right)^{2} l_{3}^{2}=0 .
$$

We have three parameters satisfying two equations, thus our kinematics depends on one free parameter. Following the notations of [11] we express $l_{1}$ in terms of the parameter $\phi$ as

$$
l_{1}=\tan (\pi / n) \tan (2 \pi / n) \tan (\phi / n) .
$$

The paramers $l_{2}$ and $l_{3}$ are then determined by (3.5) and (3.6). Note that the Wilson loop for the $\phi$ family in [11] was specified in terms of three complex variables instead of the six real variables we are using. The resulting kinematics (3.4), (3.5) is entirely equivalent to the three complex coordinates, but makes more transparent the transition between different space-time signatures in four dimensions. These transitions are necessary when one varies $\phi$ (or more generally $u_{i j}$ ) in the entire parameter space (for example when $\phi$ exceeds the value $(n-4) \pi / 2$ corresponding to the special two-dimensional regular polygon). In terms of the $l_{i}$ these transitions in signature occur when some of the previously real $l_{i}$ 's become purely imaginary, this leads to the corresponding $X_{I}$ coordinates becoming purely imaginary, giving the appropriate sign changes in (3.1).

This completes the description of the $\phi$-family geometry in terms of six-dimensional coordinates.

Below we will interpret the kinematics in terms of four dimensional Minkowski space and find the $u_{i j}$ cross-ratios, in order to compute the corresponding Wilson loops at weak coupling and compare to the strong coupling results of [11]. 
One way to get standard four-dimensional coordinates from these is to simply divide $X_{0} \ldots X_{3}$ by $X_{-1}+X_{4}$ giving

$$
x_{\mu}=\frac{1}{X_{-1}+X_{4}} X_{\mu}, \quad \mu=0 \ldots 3 .
$$

One can check that with these definitions that $x_{p p+1}^{2}:=\left(x^{(p)}-x^{(p+1)}\right)^{2}=0$ as required for a null polygon.

Having obtained the vertices of the polygon in four dimensional Minkowski space we can now find the conformally invariant cross-ratios

$$
u_{i j}=\frac{x_{i j+1}^{2} x_{i+1 j}^{2}}{x_{i j}^{2} x_{i+1 j+1}^{2}}
$$

in terms of these.

For the regular hexagon, $n=6$ the cross-ratios are

$$
u_{i i+3}=\frac{1}{4} \sec ^{2}(\phi / 3) \quad i=1,2,3,
$$

where $\phi$ varies between 0 and $3 \pi / 2$. The special value $\phi=\pi$ gives $u=1$ and corresponds to a regular polygon which can be embedded in $1+1$ dimensions. The point $\phi=0$ gives $u=1 / 4$ and corresponds to the special polygon embedded in $1+2$ dimensions. The cross-ratios $u$ become infinite at $\phi=3 \pi / 2$.

Of course the geometry is defined for any value of $\phi$ through (3.7) and one can ask what happens when $\phi$ exceeds the "extreme" point $\phi=3 \pi / 2$. What happens is immediately seen from the equation for $u_{i i+3}$, namely at $\phi=3 \pi / 2, u$ reaches infinity, makes a $u$-turn and bounces back.

For $n=8$ the cross-ratios are divided into two $Z_{n}$ invariant groups and the $\phi$ family is characterised by

$$
\begin{array}{ll}
u_{i i+3}=\frac{1}{1+\sqrt{2} \cos (\phi / 4)} & i=1, \ldots, 8 \\
u_{i i+4}=\frac{1}{2} & i=1, \ldots, 4 .
\end{array}
$$

Here $\phi$ varies between 0 (corresponding to a $1+2$ dimensional polygon) and $3 \pi$ where $u_{i i+4} \rightarrow+\infty$. It passes through the special value $\phi=2 \pi$, the special regular polygon in $1+1$ dimensions.

This time when $\phi$ passes through the "extreme" point $3 \pi$, the cross-ratio $u_{i i+3}$ goes from $+\infty$ to $-\infty$ (or equivalently the cross-ratio $1 / u_{i i+3}$ goes through zero.) In 
this way the behaviour of the cross-ratios beyond the extreme point is different from the $n=6$ case considered earlier. There is no conceptual obstruction to computing the Wilson loop at any values of $\phi$ but there are practical problems when $u<0$ as this regime can not be addressed in the fully Euclidean calculation with all two- and multi-particle invariants negative.

For $n=10$ there are three groups of cross-ratios

$$
\left\{u_{i i+3}\right\}_{i=1}^{10}, \quad\left\{u_{i i+4}\right\}_{i=1}^{10}, \quad\left\{u_{i i+5}\right\}_{i=1}^{5} .
$$

We have not attempted to find simple analytic expressions for the cross-ratios with $n \geq 10$ but there is no obstacle in doing so numerically and this will enable us to compute Wilson loops as a function of $\phi$ at $n=10$ (see section 6) and in principle beyond. We find that at $n=10, \phi$ varies between 0 and $5 \operatorname{Arccos}(-\sqrt{5} / 3)$. At the "extreme" point $\cos (\phi / 5)=-\sqrt{5} / 3, u_{i i+5}$ reaches plus infinity (and then performs a $u$-turn) while $u_{i i+4}$ goes through zero and becomes negative.

In the following sections we will compute Wilson loops for hexagonal, octagonal and decagonal $\phi$-families at one- and two-loops in perturbation theory and plot them alongside the strong coupling results.

For general $n$-polygons, $\phi$-families start at a special regular polygon in $(2+1)$ dimensions at $\phi=0$ and as one increases $\phi$ pass through another special polygon in $(1+1)$ dimensions at $\phi=\pi(n-4) / 2$ before they reach the "extreme" point where (some of) the $u$-variables become infinite. At strong coupling, the ratio of Wilson loops for all $\phi$-families is entirely determined by the free-energy expression, which is [11] a quadratic function of $\phi$

$$
A_{\text {free }}=-\frac{2}{n \pi} \phi^{2}+\text { const } .
$$

\section{Regular hexagons}

In this section we concentrate on the $\phi$-family of regular hexagons. In a later part of the section we will present the results in terms of the $\phi$ parameter, but first we want to comment on the existing results in the literature which are naturally expressed in terms of the standard cross-ratios $u$. The $\phi$-family corresponds to all $u$ 's equal and varying between $u=1 / 4$ and $u=\infty$. By extending $\phi$ to have imaginary values one can cover the full range from $u=0$ to $u=\infty$.

At weak coupling the remainder function starts at two-loops. It was first computed numerically in [7] where it was found to agree with the MHV amplitude computed 
in [6]. In [8] a formalism was developed for the numerical computation of general $n$ gon Wilson loops at the two loop level. Detailed plots of general $u$ 's are known and in particular for all $u$ 's equal we can cover the entire interval $0<u<\infty$. Furthermore analytical results have now been derived for $0<u_{1}, u_{2}, u_{3}<1$ and also in certain limits where $u_{i} \rightarrow \infty$ [26, 27]. The combined results of [8] and [27] are plotted below in figure 2 ,

The strong coupling remainder function $\mathcal{R}_{6}\left(u_{1}, u_{2}, u_{3}\right)$ has also been derived in [13] using integrable techniques. For all $u$ 's equal it takes a surprisingly simple form

$$
\mathcal{R}_{6}^{\text {strong }}(u, u, u)=\frac{\pi}{6}-\frac{1}{3 \pi} \phi^{2}-\frac{3}{8}\left(\log ^{2}(u)+2 L i_{2}(u)\right)+\text { const }
$$

where $\phi(u)$ is defined in $(\underline{3.10})$.

It is interesting to compare the dependence of the strong and weak coupling results on the kinematics. The authors of [13] made a very interesting observation. By modifying the strong coupling results (4.1) by introducing three coefficients $c_{1}, c_{2}$ and $c_{3}$,

$$
\mathcal{R}_{6}^{\mathrm{AGM}}(u, u, u)=c_{1}\left(-\frac{\pi}{6}+\frac{1}{3 \pi} \phi^{2}\right)+c_{2}\left(\frac{3}{8}\left(\log ^{2}(u)+2 L i_{2}(u)\right)\right)+c_{3},
$$

a very close match with the weak coupling remainder can be found for particular chosen values for $c_{i}$.

The constant $c_{3}$ is fixed by the collinear limit $\mathcal{R}_{6} \rightarrow \mathcal{R}_{5}$ and with a little work 11 can be found to be $c_{3}=-c_{2} \pi^{2} / 12$. We plot the combined weak coupling result and the AGM expression for $c_{1}=0.263 \pi^{3}$ and $c_{2}=0.860 \pi^{2}$.

The modified strong coupling result (4.2) and the weak coupling result at two loops, $\mathcal{R}^{(2)}$ (recall there are no one loop contributions to the remainder) are close to each other but unfortunately can never be made identical by any choice of coefficients. The strong coupling result (4.1) is not of a uniform transcendental weight. However each separate term in the modified AGM expression (4.2) has a uniform transcendental weight which can be made equal to four by choosing $c_{1} \sim \pi^{3}$ and $c_{2} \sim \pi^{2}$. It is important to note that there are no genuine weight four functions (eg no $\mathrm{Li}_{4}$ or $\log \times \mathrm{Li}_{3}$ ) in this expression. This is to be contrasted with the analytic form of the weak coupling result [26, 27] which is of transcendental weight four and where functions with intrinsic weight four do appear. For example the value of $\mathcal{R}^{(2)}$ for the

\footnotetext{
${ }^{11}$ The collinear limit brings one outside the all $u$ equal regime.
} 


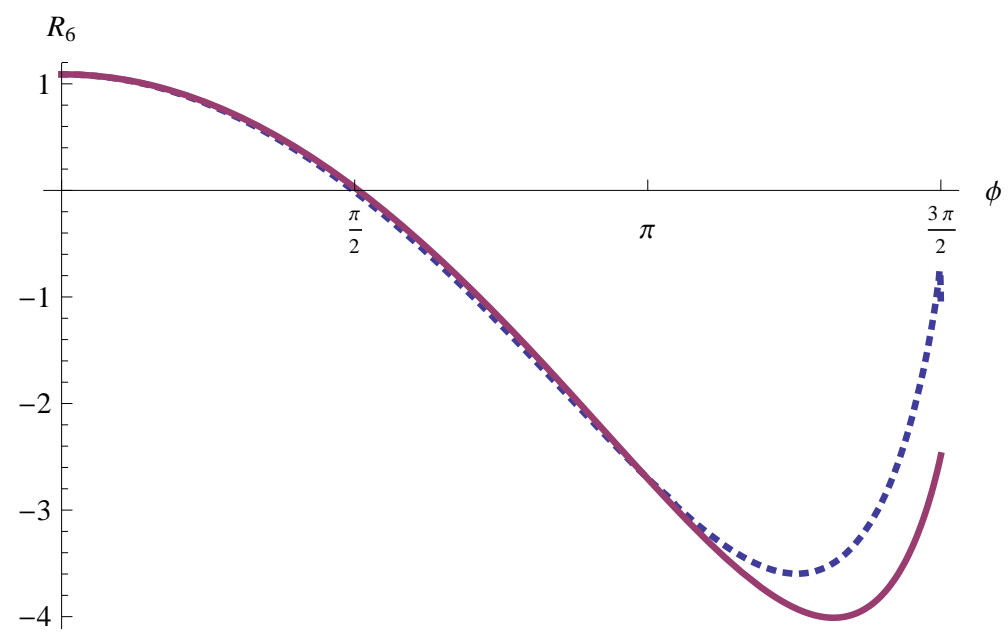

Figure 2: The remainder function for the $\phi$-family at six points. The dashed line gives the two-loop result and the solid line is the AGM modified strong coupling expression.

special regular polygon at $\phi=0($ ie $u=1 / 4)$ is [27]

$$
\begin{aligned}
\mathcal{R}_{6}^{(2)} & \left(\frac{1}{4}, \frac{1}{4}, \frac{1}{4}\right)=3 \mathrm{Li}_{2}\left(\frac{1}{3}\right) \log ^{2} 2-\frac{9}{2} \operatorname{Li}_{2}\left(\frac{1}{3}\right) \log ^{2} 3-\frac{567}{4} \mathrm{Li}_{3}\left(\frac{1}{3}\right) \log 2 \\
& +\frac{543}{4} \mathrm{Li}_{3}\left(-\frac{1}{2}\right) \log 2+\frac{567}{8} \mathrm{Li}_{3}\left(\frac{1}{3}\right) \log 3-\frac{567}{4} \mathrm{Li}_{3}\left(-\frac{1}{2}\right) \log 3+\frac{1323}{16} \zeta_{3} \log 2 \\
& +\frac{945}{32} \zeta_{3} \log 3-\frac{39}{32} \log ^{4} 2-\frac{257}{64} \log ^{4} 3+\frac{173}{8} \log 3 \log ^{3} 2+\frac{189}{8} \log ^{3} 3 \log 2-\frac{543}{16} \log ^{2} 3 \log ^{2} 2 \\
& -\frac{63}{16} \pi^{2} \log ^{2} 2-\frac{181}{64} \pi^{2} \log ^{2} 3+\frac{189}{2} \mathrm{Li}_{4}\left(\frac{1}{2}\right)+\frac{1701}{8} \operatorname{Li}_{4}\left(\frac{1}{3}\right)-\frac{543}{16} \mathrm{Li}_{4}\left(-\frac{1}{3}\right) \\
& +\frac{555}{2} \operatorname{Li}_{4}\left(-\frac{1}{2}\right)-\frac{9}{2} \mathrm{Li}_{2}\left(\frac{1}{3}\right)^{2}-\frac{567}{16} S_{2,2}\left(-\frac{1}{3}\right)-\frac{567}{4} S_{2,2}\left(-\frac{1}{2}\right)-\frac{2123 \pi^{4}}{2880} .
\end{aligned}
$$

Interestingly the value for the other special regular polygon at $\phi=\pi($ ie $u=1)$ is much simpler [8, 27]

$$
\mathcal{R}_{6}^{(2)}(1,1,1)=-\pi^{4} / 36
$$

What was the rationale for introducing different coefficients in the strong coupling result? The two terms in the remainder function have a completely different origin, the first being the free energy of an appropriate integrable system, while the second arose from subtracting the BDS expression from the cutoff area. As already discussed in section 1 the BDS expression is essentially dictated by the one-loop result and is not the object that appears naturally at strong coupling. One may hope that there is a meaning in expressing both the strong and the weak coupling results as a linear combination of certain master functions and then fitting the coefficients. But it is clear that the simple division involving $c_{1}$ and $c_{2}$ described above does not work. 


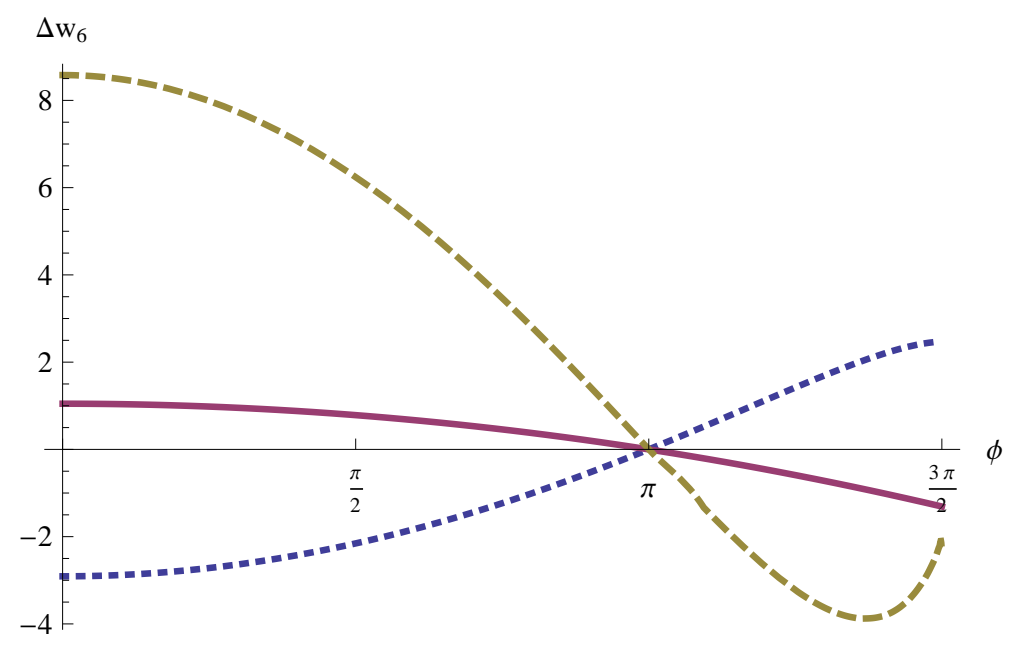

Figure 3: The Wilson loop ratio for the $\phi$-family at six points. This graph shows $\Delta w_{6}$ at one loop (dotted), two loops (dashed) and at strong coupling (solid line). The two special regular polygons are at $\phi=0$ and at $\phi=\pi$, the latter being chosen as the reference point.

At present we lack the theory giving the basis of master functions (at least at weak coupling).

We will thus concentrate on the entire Wilson loop (regularised by dividing by the reference Wilson loop as explained in section 2). Whilst the BDS remainder function was not a natural object at strong coupling, the Wilson loop ratio is natural at both strong and weak coupling. In fact at strong coupling, for the regular Wilson loops we are considering, the log of the Wilson loop ratio is just the free energy

$$
\log \left(\frac{W_{6}}{W_{6}^{\text {ref }}}\right):=w_{6}-w_{6}^{\text {ref }}=A_{\text {free }}-\text { const }=-\frac{1}{3 \pi} \phi^{2}+\frac{\pi}{3} .
$$

Here we have treated the cross-ratios for the reference Wilson loop $\tilde{u}$ as fixed. For all $\phi$-families throughout the paper we will choose all the reference Wilson loops to be the Wilson loop of the special regular polygon in $1+1$ dimensions 12 , in other words $\phi_{\text {ref }}=(n-4) \pi / 2$. At strong coupling there is only the free energy left and thus no clear reason for introducing more than one coefficient.

The Wilson loop ratio is also a natural object to compute at weak coupling. Indeed, in all computations of the remainder function, it is the Wilson loop which is computed directly and not the remainder function. The Wilson loop ratio has a

\footnotetext{
${ }^{12}$ This choice determines the constant on the right-hand side of (4.5)
} 
non-trivial contribution already at one loop

$$
w_{6}^{(1)}-w_{6}^{(1) \mathrm{ref}}=-\frac{\gamma_{K}^{(1)}}{2}\left\{\frac{3}{8}\left[\log ^{2}(u)+2 L i_{2}(1-u)\right]\right\} .
$$

This arises from the BDS expression (see Appendix).

The two loop expression $\Delta w_{6}^{(2)}(\phi):=w_{6}^{(2)}-w_{6}^{(2) \text { ref }}$ we now compute numerically as in [8]. One can always extract the remainder function from $\Delta w_{6}^{(2)}(\phi)$ by subtracting (4.6) with $\gamma_{K}^{(1)}$ replaced by $\gamma_{K}^{(2)}$.

Figure 3 gives results for $\Delta w_{6}^{(2)}(\phi)$ at one-loop, two-loops and at strong coupling. There are no coefficients, the three curves are distinct but it is interesting that the two loop result (whose analytic form consists of about 100 terms involving multiple polylogarithms) has a deceptively simple looking graph. Indeed between the two special regular polygons at $\phi=0$ and $\phi=\pi$ the function can be well approximated by a quadratic function just as at strong coupling (4.5). Furthermore, in the interval between the two special regular polygons all three contributions are almost identical up to rescaling.

We now proceed with the perturbative analysis of the $\phi$-families 13 at $n=8$ and $n=10$.

\section{Regular octagons}

The $n=8$ case is the simplest example of a polygon with $n$ divisible by four, which is a special case since the entire kinematics can not be cleanly separated into $s$ and $u$ variables. In particular for the octagon case at hand we have two cross-ratios, $\chi^{+}$and $\chi^{-}$which are made entirely from two-particle invariants. In the case of the octagon one can think of two rectangles with edges made out of two-particle invariants only, which can be embedded into the octagon. Figure 4 shows one of these rectangles, and the second one can be obtained by a cyclic relabelling $x_{i} \rightarrow x_{i+1}$ of all vertices on that figure. In terms of the variables $\chi^{ \pm}$used in [12, 28], the two rectangular cross-ratios are $\chi^{+} \chi^{-}$and $\chi^{+} / \chi^{-}$,

$$
\chi^{+} \chi^{-}=\frac{x_{35}^{2} x_{17}^{2}}{x_{13}^{2} x_{57}^{2}}=\frac{u_{15} u_{25} u_{16} u_{26}}{u_{37} u_{38} u_{47} u_{48}} \quad \frac{\chi^{+}}{\chi^{-}}=\frac{x_{24}^{2} x_{68}^{2}}{x_{46}^{2} x_{28}^{2}}=\frac{u_{48} u_{58} u_{14} u_{15}}{u_{26} u_{27} u_{36} u_{37}} .
$$

The combination $\chi^{+} / \chi^{-}$is depicted in figure 4 .

\footnotetext{
${ }^{13}$ In [1] the $\phi$-family was actually only defined within the interval between the two special regular polygons, while we continue increasing $\phi$ beyond the "extreme" point.
} 


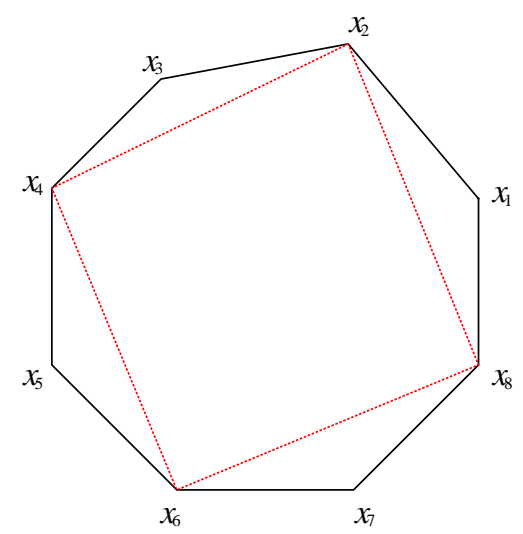

Figure 4: The cross-ratio $\chi^{+} / \chi^{-}$is a rectangle with all edges made out of two-particle invariants. The second two-particle invariant rectangle, $x_{35}^{2} x_{17}^{2} /\left(x_{13}^{2} x_{57}^{2}\right)$, is obtained by the cyclic relabelling $x_{i} \rightarrow x_{i+1}$ and corresponds to the combination $\chi^{+} \chi^{-}$.

In general we can not vary these two cross-ratios independently of $s$. However in the regular octagon case (3.11) these cross-ratios are fixed, $\chi^{+}=\chi^{-}=1$. Instead the cross-ratios we vary when we vary $\phi$ are $u_{i i+3}$ and can be varied independently of $s$.

We now consider the log of the ratio of the Wilson loops

$$
\Delta w_{8}(\phi):=w_{8}(s ; \phi)-w_{8}\left(s, \phi^{\mathrm{ref}}=2 \pi\right),
$$

where as always the reference point was chosen to be the $1+1$ dimensional special polygon. The strong coupling result for $\Delta w$ is again simply the free energy of [11] (all other contributions cancel)

$$
\Delta w_{8}^{\text {strong }}=A_{\text {free }}-\text { const }=\pi-\frac{\phi^{2}}{4 \pi} .
$$

At weak coupling we have computed the one- and two-loop contributions. The one loop result is

$$
\Delta w_{8}^{(1)}=-\gamma_{K}^{(1)}\left(\operatorname{Li}_{2}\left(1-u_{14}\right)+\log \left(\frac{u_{14}}{2}\right) \log \left(u_{14}\right)\right), \quad u_{14}=\frac{1}{1+\sqrt{2} \cos (\phi / 4)}
$$

and the two loop result is computed numerically. All three results are displayed in figure 5. Once again all three curves can be well approximated by a quadratic in the interval between the two special polygons. However, as $\phi$ reaches the extreme value the weak coupling and strong coupling results diverge.

Before we conclude this section we would like to comment on a different type of eight point kinematics, previously considered in [12,28] at strong and weak coupling 


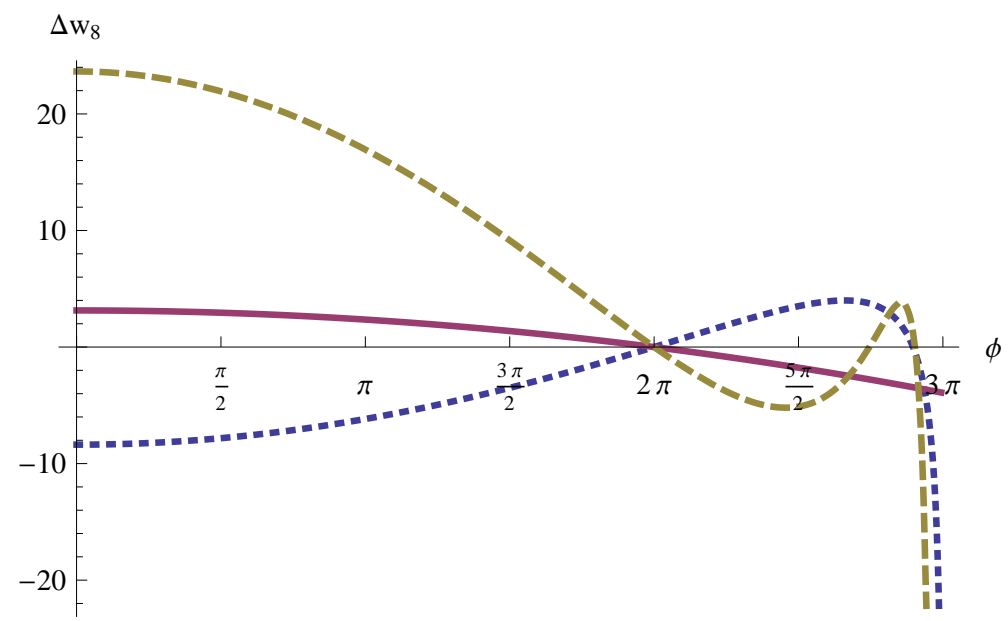

Figure 5: The Wilson loop ratio for the $\phi$-family at eight points. This graph shows $\Delta w_{8}$ at one loop (dotted), two loops (dashed) and at strong coupling (solid line). The two special regular polygons are at $\phi=0$ and at $\phi=2 \pi$, the latter being chosen as the reference point.

correspondingly. This kinematics corresponds to polygons which can be embedded in the boundary of $A d S_{3}$. In contrast to the $\phi$-kinematics discussed above, here $\chi^{+}$ and $\chi^{-}$vary while all other cross-ratios are fixed, see section 3.1 of [28] for details. This implies that in this kinematics, the only free cross-ratios, $\chi^{+}$and $\chi^{-}$are the ones which are entirely determined by the two-particle invariants, hence the ratio of Wilson loops we are considering here is trivial and thus trivially agrees between strong and weak coupling. It is curious that in this special kinematics the two-loop remainder numerically matched the strong coupling counter-part up to an overall rescaling [28]. It is tempting to compare this situation to results for $n<6$ where the ratio is also trivial as there are no multi-particle invariants. These are the cases where strong and weak coupling results also agree as they are given by the BDS expression.

\section{$6 \quad$ Regular decagons}

The final set of computations which we have performed for the $\phi$-family is for $n=10$ regular polygons and our strategy of dividing by the reference area works without exceptions. The strong coupling result for $\Delta w_{10}$ is once again determined by the free energy of reference [11] and is quadratic in $\phi$. The one loop result can be determined from the BDS expression, and the two-loop result we have computed. These three contributions are plotted alongside each other in figure 6. As always, the one-loop result should be multiplied by $a$, the two-loop result by $a^{2}$ and the strong coupling result by $\sqrt{2 a}$. 


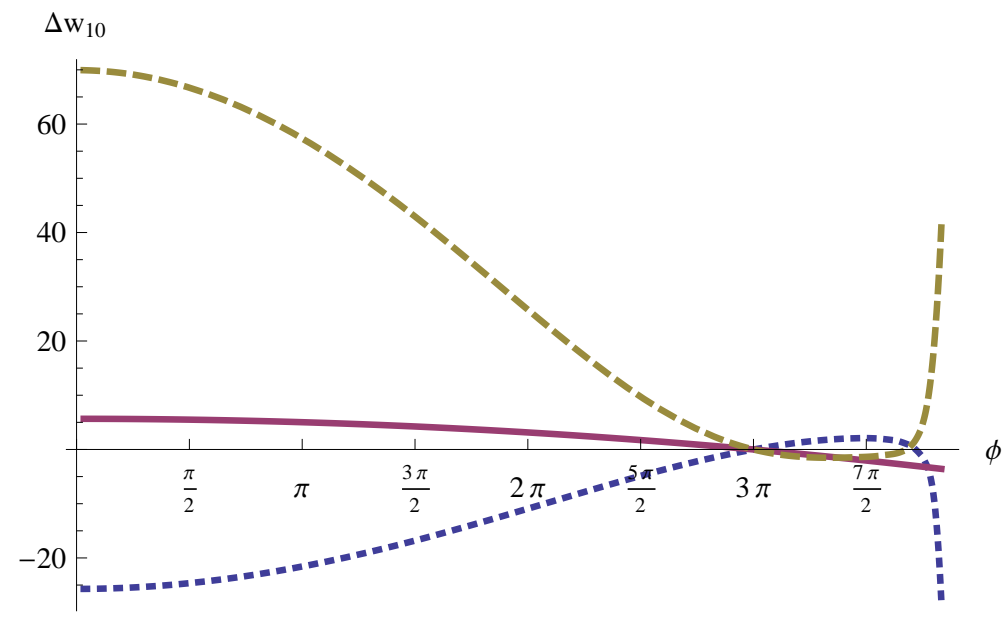

Figure 6: The Wilson loop ratio for the $\phi$-family at eight points. This graph shows $\Delta w_{10}$ at one loop (dotted), two loops (dashed) and at strong coupling (solid line). The two special regular polygons are at $\phi=0$ and at $\phi=3 \pi$, the latter being chosen as the reference point.

Once again between the two special polygons at $\phi=0$ and $\phi=3 \pi$ there is a similarity between the three curves, though the two loop curve is starting to visibly deviate from the quadratic form. Beyond $3 \pi$ and especially as $\phi$ approaches the extreme point, the weak coupling results start to diverge, whilst the strong coupling result does not.

To summarise, in this paper we have proposed a new finite, regularisation independent and conformally invariant way to characterise the Wilson loops and amplitudes in both weak and strong coupling regimes. As an application, we have computed Wilson loops for regular polygons, up to $n=10$ and have compared our weak coupling results to the recently derived strong coupling expressions of [11].

\section{Acknowledgements}

It is a pleasure to thank Babis Anastasiou, Andreas Brandhuber, Patrick Dorey, Claude Duhr, Bill Spence and Gabriele Travaglini for discussions at various stages of this work. VVK acknowledges a Leverhulme Research Fellowship. 


\section{Appendix}

\section{A BDS}

For the convenience of the reader we here define the BDS expressions appearing in section 1. In the context of MHV amplitudes in dimensional regularisation with $D=4-2 \epsilon$ we have [10]

$$
(B D S)_{n}=\sum_{L=1}^{\infty} a^{L} f^{(L)}(\epsilon) \mathcal{M}_{n}^{(1)}(L \epsilon)+C(a) .
$$

Here $\mathcal{M}_{n}^{(1)}(\epsilon)$ is the one loop amplitude, $a$ is the coupling constant $a=\left[g^{2} N /\left(8 \pi^{2}\right)\right]\left(4 \pi e^{-\gamma}\right)^{\epsilon}$ and

$$
f^{(L)}(\epsilon):=f_{0}^{(L)}+f_{1}^{(L)} \epsilon+f_{2}^{(L)} \epsilon^{2} .
$$

The $f_{i}^{(L)}$ are numbers, in particular, $f_{0}^{(L)}=\gamma_{K}^{(L)} / 4$, where $\gamma_{K}$ is the cusp anomalous dimension,

$$
\gamma_{K}(a)=\sum_{L=1}^{\infty} a^{L} \gamma_{K}^{(L)}, \quad \gamma_{K}^{(1)}=4, \quad \gamma_{K}^{(2)}=-4 \zeta_{2},
$$

and $C(a)$ is a (coupling dependent) constant starting from two loops, $C^{(2)}=-\zeta_{2}^{2} / 2$.

The one-loop amplitude contains an infrared divergent part and a finite part

$$
\begin{aligned}
\mathcal{M}_{n}^{(1)}(\epsilon) & =-\frac{1}{2 \epsilon^{2}} \sum_{i=1}^{n}\left(\frac{-s_{i}}{\mu^{2}}\right)^{-\epsilon}+F_{n}^{(1)}(\epsilon), \\
F_{n}^{(1)}(0) & =\frac{1}{2} \sum_{i=1}^{n} g_{n, i},
\end{aligned}
$$

where

$$
g_{n, i}=-\sum_{r=2}^{[n / 2]-1} \ln \left(\frac{-t_{i}^{[r]}}{-t_{i}^{[r+1]}}\right) \ln \left(\frac{-t_{i+1}^{[r]}}{-t_{i}^{[r+1]}}\right)+D_{n, i}+L_{n, i}+\frac{3}{2} \zeta_{2},
$$

and $t_{i}^{[r]}:=\left(p_{i}+\cdots+p_{i+r-1}\right)^{2}$ are the kinematical invariants, $t_{i}^{[2]}=s_{i}$ and $t_{i}^{[r>2]}$ are multi-particle invariants. The functions $D_{n, i}$ and $L_{n, i}$ for even values of $n$ are [29]

$$
\begin{aligned}
D_{2 m, i} & =-\sum_{r=2}^{m-2} \operatorname{Li}\left(1-\frac{t_{i}^{[r]} t_{i-1}^{[r+2]}}{t_{i}^{[r+1]} t_{i-1}^{[r+1]}}\right)-\frac{1}{2} \operatorname{Li}\left(1-\frac{t_{i}^{[m-1]} t_{i-1}^{[m+1]}}{t_{i}^{[m]} t_{i-1}^{[m]}}\right), \\
L_{2 m, i} & =-\frac{1}{4} \ln \left(\frac{-t_{i}^{[m]}}{-t_{i+m+1}^{[m]}}\right) \ln \left(\frac{-t_{i+1}^{[m]}}{-t_{i+m}^{[m]}}\right) .
\end{aligned}
$$


It is clear from (A.1,A.4 that to all orders in perturbation theory the divergent part of the BDS expression depends on the kinematics only through two-particle invariants, and the finite part is given by

$$
F_{n}^{\mathrm{BDS}}(a)=\frac{1}{4} \gamma_{K}(a) F_{n}^{(1)}(0)+C(a)
$$

The BDS expression for the Wilson loop, $(B D S)_{n}^{W L}$ has the same form as that of the amplitude, $(B D S)_{n}$ except that the one loop amplitude $\mathcal{M}_{n}^{(1)}$ is substituted by the one loop expression for the Wilson loop $W_{n}^{(1)}$ and the coefficient functions $f(\epsilon)$ are different, $f_{W L}(\epsilon)$,

$$
\begin{aligned}
f^{(1)}(\epsilon) & =1 & f^{(2)}(\epsilon) & =-\zeta_{2}-\zeta_{3} \epsilon-\zeta_{4} \epsilon^{2} \\
f_{W L}^{(1)}(\epsilon) & =1 & f_{W L}^{(2)}(\epsilon) & =-\zeta_{2}+7 \zeta_{3} \epsilon-5 \zeta_{4} \epsilon^{2} .
\end{aligned}
$$

The one loop contribution to the Wilson loop agrees with the one loop amplitude up to a constant [2] for any $n$ [3]

$$
W_{n}^{(1)}=\mathcal{M}_{n}^{(1)}-n \frac{\pi^{2}}{12}
$$

This statement is of course the one loop manifestation of the Wilson loop/amplitude duality. At higher loops as is by now well known, the BDS expressions for both the amplitude and the Wilson loop need to be extended by introducing remainder functions as in (1.2). The statement of the duality beyond one loop can be characterised by the equality of the remainder functions (see equation (1.3).

\section{References}

[1] L. F. Alday and J. Maldacena, Gluon scattering amplitudes at strong coupling, JHEP 0706 (2007) 064, 0705.0303 [hep-th].

[2] J. M. Drummond, G. P. Korchemsky and E. Sokatchev, Conformal properties of four-gluon planar amplitudes and Wilson loops, Nucl. Phys. B 795 (2008) 385, 0707.0243 [hep-th].

[3] A. Brandhuber, P. Heslop and G. Travaglini, MHV Amplitudes in N=4 Super Yang-Mills and Wilson Loops, Nucl. Phys. B 794 (2008) 231, 0707.1153 [hep-th].

[4] J. M. Drummond, J. Henn, G. P. Korchemsky and E. Sokatchev, On planar gluon amplitudes/Wilson loops duality, Nucl. Phys. B 795 (2008) 52, 0709.2368 [hep-th]. 
[5] J. M. Drummond, J. Henn, G. P. Korchemsky and E. Sokatchev, Conformal Ward identities for Wilson loops and a test of the duality with gluon amplitudes, Nucl. Phys. B 826 (2010) 337 [arXiv:0712.1223 [hep-th]].

[6] Z. Bern, L. J. Dixon, D. A. Kosower, R. Roiban, M. Spradlin, C. Vergu and A. Volovich, The Two-Loop Six-Gluon MHV Amplitude in Maximally Supersymmetric Yang-Mills Theory, Phys. Rev. D 78, 045007 (2008), 0803.1465 [hep-th].

[7] J. M. Drummond, J. Henn, G. P. Korchemsky and E. Sokatchev, Hexagon Wilson loop = six-gluon MHV amplitude, Nucl. Phys. B 815 (2009) 142, 0803.1466 [hep-th] .

[8] C. Anastasiou, A. Brandhuber, P. Heslop, V. V. Khoze, B. Spence and G. Travaglini, Two-Loop Polygon Wilson Loops in N=4 SYM, JHEP 0905 (2009) $115,0902.2245$ [hep-th].

[9] C. Anastasiou, Z. Bern, L. J. Dixon and D. A. Kosower, Planar amplitudes in maximally supersymmetric Yang-Mills theory, Phys. Rev. Lett. 91 (2003) 251602, hep-th/0309040.

[10] Z. Bern, L. J. Dixon and V. A. Smirnov, Iteration of planar amplitudes in maximally supersymmetric Yang-Mills theory at three loops and beyond, Phys. Rev. D 72 (2005) 085001, hep-th/0505205.

[11] L. F. Alday, J. Maldacena, A. Sever and P. Vieira, Y-system for Scattering Amplitudes, arXiv:1002.2459.

[12] L. F. Alday and J. Maldacena, Null polygonal Wilson loops and minimal surfaces in Anti-de-Sitter space, JHEP 0911, 082 (2009) [arXiv:0904.0663 [hep-th]].

[13] L. F. Alday, D. Gaiotto and J. Maldacena, Thermodynamic Bubble Ansatz, arXiv:0911.4708.

[14] Y. Hatsuda, K. Ito, K. Sakai and Y. Satoh, Thermodynamic Bethe Ansatz Equations for Minimal Surfaces in $A d S_{3}$, arXiv:1002.2941 [hep-th].

[15] L. F. Alday, J. M. Henn, J. Plefka and T. Schuster, Scattering into the fifth dimension of $N=4$ super Yang-Mills, JHEP 1001, 077 (2010) arXiv:0908.0684 [hep-th]].

[16] Z. Bern, M. Czakon, D. A. Kosower, R. Roiban and V. A. Smirnov, Two-loop iteration of five-point $N=4$ super-Yang-Mills amplitudes, Phys. Rev. Lett. 97 (2006) 181601, hep-th/0604074.

[17] V. Del Duca, C. Duhr and E. W. Nigel Glover, The five-gluon amplitude in the high-energy limit, JHEP 0912 (2009) 023 [arXiv:0905.0100 [hep-th]]. 
[18] A. H. Mueller, On The Asymptotic Behavior Of The Sudakov Form-Factor, Phys. Rev. D 20 (1979) 2037.

[19] J. C. Collins, Algorithm To Compute Corrections To The Sudakov Form-Factor, Phys. Rev. D 22 (1980) 1478.

[20] A. Sen, Asymptotic Behavior Of The Sudakov Form-Factor In QCD, Phys. Rev. D 24 (1981) 3281.

[21] L. Magnea and G. Sterman, Analytic continuation of the Sudakov form-factor in QCD, Phys. Rev. D 42 (1990) 4222.

[22] J. M. Drummond, J. Henn, G. P. Korchemsky and E. Sokatchev, Dual superconformal symmetry of scattering amplitudes in $N=4$ super-Yang-Mills theory, Nucl. Phys. B 828 (2010) 317 [arXiv:0807.1095 [hep-th]].

[23] A. Brandhuber, P. Heslop and G. Travaglini, A note on dual superconformal symmetry of the N=4 super Yang-Mills S-matrix, Phys. Rev. D 78 (2008) 125005 arXiv:0807.4097 [hep-th]].

[24] A. Brandhuber, P. Heslop and G. Travaglini, Proof of the Dual Conformal Anomaly of One-Loop Amplitudes in N=4 SYM, JHEP 0910 (2009) 063 arXiv:0906.3552 [hep-th]].

[25] S. Abel, S. Forste and V. V. Khoze, Scattering amplitudes in strongly coupled $N=4 S Y M$ from semiclassical strings in AdS, JHEP 0802 (2008) 0420705.2113 [hep-th].

[26] V. Del Duca, C. Duhr and V. A. Smirnov, An Analytic Result for the Two-Loop Hexagon Wilson Loop in $N=4 S Y M$, arXiv:0911.5332,

[27] V. Del Duca, C. Duhr and V. A. Smirnov, The Two-Loop Hexagon Wilson Loop in $N=4 S Y M$, arXiv:1003.1702.

[28] A. Brandhuber, P. Heslop, V. V. Khoze and G. Travaglini, Simplicity of Polygon Wilson Loops in N=4 SYM, JHEP 1001, 050 (2010) [arXiv:0910.4898].

[29] Z. Bern, L. J. Dixon, D. C. Dunbar and D. A. Kosower, One Loop N Point Gauge Theory Amplitudes, Unitarity And Collinear Limits, Nucl. Phys. B 425 (1994) 217 , hep-ph/9403226. 